\title{
Future Directions in Metastatic Castration Resistant Prostate Cancer (mCRPC): Clinical Rationale and Use of New-Generation Hormonal Therapies
}

\author{
Paolo Grassi, Elena Verzoni, Rosanna Montone, Isabella Testa, Enrico Garanzini, Pamela Biondani, \\ Sara Pusceddu, Filippo de Braud, Giuseppe Procopio*
}

Department of Medical Oncology, Fondazione IRCCS Istituto Nazionale Tumori, Milan, Italy.

Email: " giuseppe.procopio@istitutotumori.mi.it

Received February $28^{\text {th }}, 2013$; revised March 27 $7^{\text {th }}, 2013$; accepted April $4^{\text {th }}, 2013$

Copyright (C) 2013 Paolo Grassi et al. This is an open access article distributed under the Creative Commons Attribution License, which permits unrestricted use, distribution, and reproduction in any medium, provided the original work is properly cited.

\begin{abstract}
Several recent studies in mCRPC have identified the mechanisms of tumoral growth after the disease becomes unresponsive to standard hormonal therapy. These studies have highlighted the importance of residual intratumoral androgens in maintaining androgen receptor (AR) transcriptional activity in mCRPC [1]. Based on this findings, it has been possible to develop drugs, blocking the key enzyme in the biosynthesis of androgens through the inhibition of cytochrome p450 17 (CYP17) such as Abiraterone Acetate (AA) and drugs which directly target the AR including Enzalutamide (E) and Orteronel. Before this new knowledge, mCRPC treatment benefited from chemotherapy with taxanes. Recently a new taxane, Cabazitaxel (C), was approved in second line setting in association with prednisone. Retrospective analyses have tried to clarify the current role of chemotherapy in mCRPC patients and the right chemotherapy sequence of use of chemotherapy compared to new hormonal agents. Moreover, it would be important to address changes in the endpoints used in clinical trials, based on the stage of disease including the presence tumor-related symptoms, in order to identify the right therapeutic strategy.
\end{abstract}

Keywords: Metastatic Castration Resistant Prostate Cancer; Abiraterone; Cabazitaxel; Enzalutamide

\section{Introduction}

Prostate cancer is the most common cancer in males [2] and the second leading cause of cancer-related death in the western countries [3]. Although diagnosis is mainly made at an early stage and many patients with prostate cancer may be considered cured after loco-regional approach, a small percentage of them experience disease recurrence and will develop metastases. Hormonal therapy with analogues of luteinizing hormone-releasing hormone (LHRH analogues) and antiandrogens is the initial approach for prostate cancer patients undergoing medical treatment. However, even if hormonal treatment is effective in more than $90 \%$ of patients response to treatment is limited over time. Until recently, the lack of response to hormonal manipulation was defined as a resistance stage and used to be considered irreversible. Currently, patients not responding to standard hormonal therapy are defined as castration resistant (CRPC) and new acquisitions on

${ }^{*}$ Corresponding author. the disease pathology lead to the development of new molecules effective in advanced stage. Prostate cancer growth depends on the ratio of proliferating cells over those dying. Androgens are the main regulators of this process by either stimulating proliferation or inhibiting apoptosis [4]. In fact it is now established that tumor cells are able to proliferate even with testosterone levels below the castration threshold $(<50 \mathrm{ng} / \mathrm{mL})$. Despite administration of androgen depleting therapies, activated AR signaling is a common feature in CRPC due to AR gene amplification, gene mutation, increased AR expression or increased androgen biosynthesis in prostate tumor cells. This is supported by the evidence of higher levels of testosterone derivatives in cancer tissue compared to plasma [5]. The androgen-signaling pathway plays an important role in the development and progression of prostate cancer and androgen ablation is a mainstay of therapy for this disease. Abiraterone acetate is the first highly selective, irreversible CYP-17 inhibitor, preventing androgen conversion in testis and adrenal glands and suppressing 
de novo androgen production in prostate tumor cells. Encouraging results deriving from clinical research are the basis for the development of drugs having the interaction of androgens with androgen receptor as a main target in CRPC. The recent approval of enzalutamide for postdocetaxel mCRPC provides another valuable opportunity for long term patient treatment. Before these results, the golden standard for symptomatic metastatic prostate cancer was represented by chemotherapy. Although there was no evidence of clinical benefit in anticipating docetaxel treatment, the lack of therapeutic alternatives led to anticipate chemotherapy in metastatic patients before the onset of symptoms or other risk factors (anemia, visceral metastases) [6]. The current role of chemotherapy is to be clarified, considering a new treatment opportunity such as cabazitaxel, a new member of the family of taxane that has been shown to increase survival in patients with mCRPC pretreated with docetaxel. Recently published data on AA in chemotherapy naive setting, showed a statistically significant improvement in progression-free survival compared to placebo plus prednisone, and longer overall survival in men with metastatic castration-resistant prostate cancer [7]. Based on these data, it may be useful to start thinking about the right sequential treatment strategy based on the new objectives that should acquire priority at different stages of CRPC.

\section{Second-Line Therapy: New Options}

The pivotal trial of docetaxel [8] demonstrated in 2004 for the first time that chemotherapy can increase survival in patients with metastatic prostate cancer and this still represent the standard of care in the first line setting. Until recently there has been no standard treatment after docetaxel failure, even though a few drugs such as mitoxantrone and ketoconazole are administered as a palliative treatment [9]. In the absence of proven benefit in terms of overall survival also docetaxel rechallenge was considered for second line treatment for patients with a good response to the first line chemotherapy. In fact, several retrospective or single arm studies showed a PSA response rate of $38 \%-48 \%$ with an overall survival of up to 16 months $[10,11]$. Di Lorenzo et al. performed a small phase II study of docetaxel rechallenge in patients who showed either response or stable disease during first-line docetaxel. Their results were less favorable than previous studies, showing a PSA response rate of $24.5 \%$ and an overall survival of 13 months [12]. Several studies have recently been published showing a survival advantage with new drugs for second line treatment of mCRPC.

\subsection{Abiraterone Acetate}

The emerging evidence on the activity of low level tes- tosterone in CRPC have led to the development of AA, an oral drug that has been shown to be active after failure of docetaxel-based chemotherapy. Abiraterone acetate is a potent, irreversible inhibitor of cytochrome P450-17 blocking the enzymatic activity of 17alpha-monooxygenase (CYP17alpha-hydrolase/CYP17, 20 lyase complex), a member of the cytochrome $\mathrm{P} 450$ family that catalyzes the 17alpha-hydroxylation of steroid intermediates involved in the synthesis of testosterone. [13] The suppression of testosterone synthesis caused by this agent is achieved at testicular, adrenal gland and tumor cells level. The efficacy and tolerability of AA associated with prednisone have been shown in several phase 1 and phase 2 studies $[14,15]$. The dose of $1000 \mathrm{mg}$ daily has been identified and used in the clinical development. The phase 3 pivotal study of AA in combination with prednisone in symptomatic mCRPC patients after docetaxel failure enrolled 1195 patients. The patients treated in the study may have also received two lines of chemotherapy and had an ECOG performance status between 0 and 2. For the first time a second-line agent other than chemoterapy showed a significant improvement in overall survival in mCRPC patients ( 15.8 vs 11.2 months HR $0.65,95 \%$ CI 0.54 $0.77 ; \mathrm{p}<0.0001)$. Moreover, AA showed a benefit in all secondary endpoints: time to PSA progression, PSA response rate, radiographic progression-free survival (rPFS) and objective response rate. Since the major cause of morbidity in this setting is represented by bone metastases, affecting more than $90 \%$ mCRPC patients, the bone related endpoints deserve particular emphasis. A clinically significant improvement in pain palliation and a statistically significant improvement in the time to skeletal related events have been recorded with AA treatment [16]. In patients with clinically significant pain at baseline, AA and prednisone had a positive statistically significant impact on palliation $(45.0 \%$ vs $28.8 \% \mathrm{p}=$ 0.0005 ), with a median time to palliation of 5.6 vs 13.7 months. In the overall trial population the median time to occurrence of first skeletal-related event was significantly longer with AA and prednisone compared to prednisone alone ( 25.0 vs 20.3 months $p=0.0001)$. In the same study a significant benefit of AA on patient reported outcome, quality of life and fatigue was also demonstrated [17]. The safety profile is another important feature for a drug in this setting. Most common grade 1 - 2 adverse events were fatigue, back pain, nausea, constipation an arthralgia, which were very similar to the control arm. Adverse events associated with elevated mineralocorticoid level due to CYP17 blockade (fluid retention, edema, hypokalemia and hypertension) as well as cardiac disorders and liver function test abnormalities were more common in AA group than the control group. These events were mainly grade $1-2$ with grade $3<3 \%$. 


\subsection{Cabazitaxel}

As already underlined, taxanes are the only chemotherapeutic agents effective in the treatment of symptomatic metastatic prostate cancer. However, their high affinity for the protein substrate of the multidrug resistance (MDR) complex represents a potential limitation [18]. Cabazitaxel is a new taxane registered for the second line treatment of mCRPC with the ability to overcome mechanisms of resistance [19] and to improve survival. In fact, in the TROPIC study, median overall survival was 15.1 months versus 12.7 months. This result corresponds to a $30 \%$ relative risk of death reduction (HR $0.70,95 \% \mathrm{CI}$ $0.59-0.83 p<0.0001)$ [20]. Patients in the experimental arm had a longer progression-free survival (PFS) compared to control arm (2.8 vs 1.4 months) and the tumor response rate, PSA response, time to tumor progression and time to PSA progression were significantly higher with cabazitaxel compared to mitoxantrone. Quality of life data are similar in the two study arm due to the use of a well recognized palliative agent in the control arm. The safety profile is consistent with taxane agents, being myelosuppression the most relevant hematological side effect. In particular in the pivotal trial, grade $\geq 3$ neutropenia occurred in $81 \%$ of treated cases, suggesting that treatment with colony stimulating factors may be necessary in patients older than 65 years and with risk factors for febrile neutropenia.

\subsection{Enzalutamide}

Enzalutamide is a new drug with a potent and selective mechanism of action directed against the AR, recognizing the central role of this target in the development of advanced CRPC. Enzalutamide blocks androgen signaling by directly binding the AR and inhibiting either nuclear translocation or coactivator recruitment of the ligand receptor [21]. The pivotal study of E enrolled symptomatic mCRPC patients after docetaxel-based chemotherapy failure and showed an improvement in overall survival (18.4 vs 13.6 months, HR for death in the $\mathrm{E}$ group, $0.63 ; 95 \% \mathrm{CI}, 0.53$ to $0.75 ; \mathrm{p}<0.001$ ) with a manageable safety profile. The pivotal clinical trial PREVAIL in asymptomatic or mildly-symptomatic chemo-naive patients is ongoing. As for AA, the statistically significant benefit in overall survival was observed in all subgroups analyzed (ECOG PS, pain score at baseline, biochemical or radiographic progression at baseline and visceral metastatic disease). The superiority of $\mathrm{E}$ over placebo was shown for all secondary endpoints, including PSA response rate $(54 \%$ vs $2 \%, p<0.001)$, the soft tissue response rate $(29 \%$ vs $4 \%, \mathrm{p}<0.001)$, FACT-P quality of life $(43 \%$ vs $18 \%, p<0.001)$, radiographic PFS $(8.3$ mo vs 2.9 , mo HR 0.40 ), time to first skeletal event (16.7 vs 13.3 , HR 0.69 ) and time to PSA progression ( 8.3 vs 3.0, months HR 0.25) [22]. In the pivotal study, fatigue was the most common side effect. There was an increased incidence of headache in patients treated with $\mathrm{E}$, and this side effect may be associated with an increased risk of seizures [23]. The effectiveness of all these agents also in terms of improvement of bone related endpoints and quality of life, confirm the importance of inhibiting different pathways underlying tumor growth and progression. We believe that further prospective studies to investigate the right sequence of the various agents available are strongly warranted.

\section{Sequencing: Data and Hypotheses}

Until recently, mCRPC patients were considered unresponsive to hormonal therapy. In the era of new drugs the approach to the disease has dramatically changed and the real concern is now focused on drug sequencing and the role of chemotherapy so far considered the standard of care. As a demonstration of this unmet medical need, a few reports have tried to address this issue in an attempt to retrospectively explore the best sequencing: in the first one [24], the authors presented the results of a retrospective study investigating the activity of docetaxel in mCRPC patients previously treated with AA using biochemical and radiological criteria according to Prostate cancer Working Group 2 (PCWG2). Overall 35 patients who received docetaxel after AA failure were considered for this analysis. All patients had received AA within phase I or II trials at different doses. The authors reported a lower activity of docetaxel post-AA with no responses observed in patients refractory to AA ( 8 cases). Nine patients $(26 \%)$ experienced an early disease progression during treatment with docetaxel while a PSA $>50 \%$ response was documented in 9 cases $(26 \%)$ and a $30 \%$ PSA reduction was observed in 13 cases (37\%). The median time to PSA progression was 4.6 months, and median overall survival was 12.5 months. The authors' conclusions were that the sequential use of docetaxel after AA failure may be associated to reduced efficacy. The authors' opinion is that the potential cross resistance may be due to the interaction between taxanes and the AR and to the increased level of intratumoral androgens that would be observed after AA suspension. The lower activity of docetaxel may be explained by the fact that this assessment was made with patients with advanced disease and in the absence of valid therapeutic alternatives, as is the case of the patients included in phase I trials. In this analysis patients still responding to treatment with $\mathrm{AA}$ were excluded. Another retrospective analysis performed to evaluate the right drug sequence was carried out by Albiges et al. [25] In this abstract, 38 pts treated with cabazitaxel after AA were analyzed. An average of 4 
cycles of C (range 1 - 9) were given, $85 \%$ of patients received granulocyte-colony stimulating factor prophylaxis from cycle 1 , and 15 pts $(39 \%)$ were receiving cabazitaxel at the time of safety analysis. Out of 38 patients, 16 patients discontinued treatment before completing the full course and 15 of these patients showed disease progression. No major toxicities were noticed. Of 32 patients with PSA data available, $18(56 \%)$ patients had a $50 \%$ or greater PSA decline, 5 patients $(15 \%)$ showed a partial response. Authors concluded that $\mathrm{C}$ seems to be active and well tolerated in mCRPC patients refractory to AA. Nevertheless this data do not provide any evidence for cross-resistance between these two agents. Due to heterogeneity of mCRPC patients and considering the role that androgens and AR play on disease progression, we think that early administration of new hormonal therapy could be more useful for the subgroup of patients with a long-lasting response to previous standard hormonal treatments. Conversely, in the presence of poorly differentiated, neuroendocrine and aggressive disease, not to delay the start of chemotherapy would be more appropriate. Visceral metastases are considered an important poor risk factor in mCRPC patients. Recent data regarding treatment with AA in this subgroup of patients were presented during the last ASCO GU meeting. The absolute OS benefit favoring AA was similar in patients with visceral and non-visceral disease. AA has substantial antitumor activity and provides clinical benefit, including statistically significant improvement in objective response, PSA response, and rPFS in post-docetaxel mCRPC patients with visceral disease. The phase 3 study for asymptomatic or midly-symptomatic docetaxel-naive patients showed that AA is also active in this setting. The analysis of patients treated in second line in this randomized study with docetaxel would certainly be more informative. For the same reason, considering the effectiveness of $\mathrm{E}$ in postchemotherapy patients, the phase 3 study PREVAIL was designed to test the efficacy of this antiandrogen before docetaxel. The possible mechanism of cross-resistance suggested by the authors highlights the importance of the AR interactions for progression of castration-resistant disease. Therefore, it is currently impossible to identify an optimal sequence of treatment suitable for all mCRPC patients. In our opinion, the physician should optimize the use of different agents on the basis of both the patients and disease characteristics, such as age, comorbidity, life expectancy and concomitant therapies. Finally, a retrospective analysis of the sequence with both AA and E, has been explored. In particular, two analyses presented during the ESMO Meeting 2012 have shown only modest antitumor activity of the AA when used after docetaxel failure and the noncross-resistance between the two agents, [26] suggesting that the decreased response to one of the two agents does not exclude the efficacy of the other one. In conclusion, there is a lack of clinical and preclinical data available to support the choice of a specific sequence of drugs for the treatment of mCRPC. In our opinion, overall PFS should be considered, analyzing the PFS reported with each agent.

\section{New Objectives in the Treatment of CRPC}

There is no standard therapy for asymptomatic or mildlysymptomatic mCRPC patients. In fact, the current EAU guidelines recommend the use of second-line hormonal therapy with antiandrogens or glucocorticoids such as prednisone/prednisolone for these patients. Based on this data, the comparator used for the phase 3 COUAA 302 trial with AA plus prednisone in the pre-chemotherapy setting was the low-dose prednisone. Historically composite endpoints were used to evaluate the efficacy in this patient population, the assessment of the PSA response and the clinical impact in terms of palliation of symptoms [27]. The choice of PFS in association with OS in the COUAA 302 study was influenced by the need to have an endpoint also available for an independent committee. The median radiographic PFS was 16.5 months (HR 0.53 $\mathrm{p}<0.0001)$ in the AA plus prednisone arm and 8.3 months in the prednisone arm [28]. Over a median follow up of 22.2 months, OS was improved in the AA plus prednisone arm (median not reached, vs 27.2 months for prednisone alone HR 95\% CI: 0.74, $\mathrm{p}=0.0017$ ) but did not cross the efficacy boundary. Abiraterone plus prednisone showed superiority over prednisone alone regarding time to initiation of cytotoxic chemotherapy, opiate use for cancer-related pain, PSA progression, and decline in performance status. This data are very important if we consider the stage of the disease, since the extension of both time to chemo-therapy and deterioration of performance status affect the natural history of mCRPC. Based on these results and the growing development of new drugs, we think that the approach to prostate cancer is changing. With these new therapeutical options, we could change the perception of the disease in MCRPC patients, improving quality of life, pain and decreasing the side effects related to cytotoxic chemotherapy. The aim of treatment varies depending on the stage of the disease and, the PSA levels as a marker in the advanced disease seem to have no relevance. Overall survival remains the main goal but in the assessment of individual drug efficacy the PFS still represents a valid surrogate for OS since it can be influenced by the effect of sequential treatments.

\section{REFERENCES}

[1] D. J. Bedoya and N. Mitsiades, "Clinical Appraisal of Abiraterone in the Treatment of Metastatic Prostatic Can- 
cer: Patient Considerations, Novel Opportunities, and Future Directions," OncoTargets and Therapy, Vol. 2013, No. 6, 2013, pp. 9-18. doi:10.2147/OTT.S24941

[2] Office for National Statistics, "Cancer Statistics Registrations: Registrations of Cancer Diagnosed in 2008, England," MB1: No. 39 Edition, Office for National Statistics, London, 2010.

[3] Office for National Statistics, "Statistical Bulletin: Death Registrations by Cause in England and Wales, 2009," Office for National Statistics, London, 2010.

[4] B. J. Feldman and D. Feldman, "The Development of Androgen-Independent Prostate Cancer," Nature Reviews: Cancer, Vol. 1, No. 1, 2001, pp. 34-45. doi:10.1038/35094009

[5] R. B. Montgomery and E. A. Mostaghel, "Maintenance of Intratumoral Androgens in Metastatic Prostate Cancer: A Mechanism for Castration-Resistant Tumor Growth," Cancer Research, Vol. 68, No. 11, 2008, pp. 4447-4454. doi:10.1158/0008-5472.CAN-08-0249

[6] A. J. Armstrong and I. F. Tannock, "The Development of Risk Groups in Men with Metastatic Castration-Resistant Prostate Cancer Based on Risk Factors for PSA Decline and Survival," European Journal of Cancer, Vol. 46, No. 3, 2010, pp. 517-525. doi:10.1016/j.ejca.2009.11.007

[7] C. J. Ryan, M. R. Smith, et al., "Abiraterone in Metastatic Prostate Cancer without Previous Chemotherapy," The New England Journal of Medicine, Vol. 368, No. 2, 2013, pp. 138-148. doi:10.1056/NEJMoa1209096

[8] I. F. Tannock, R. De Wit, et al., "Docetaxel plus Prednisone or Mitoxantrone plus Prednisone," The New England Journal of Medicine, Vol. 351, 2004, pp. 1502-1512. doi:10.1056/NEJMoa040720

[9] I. F. Tannock, I. Osoba, et al., "Chemotherapy with Mitoxantrone plus Prednisone or Prednisone Alone for Symptomatic Hormone-Resistant Prostate Cancer: A Canadian Randomized Trial with Palliative End Points," Journal of Clinical Oncology, Vol. 14, No. 6, 1996, pp. 1756-1764.

[10] J. C. Eymard, S. Oudard, et al., "Docetaxel Reintroduction in Patients with Metastatic Castration-Resistant Docetaxel-Sensitive Prostate Cancer: A Retrospective Multicentre Study," BJU International, Vol. 106, No. 7, 2010, pp. 974-978. doi:10.1111/j.1464-410X.2010.09296.X

[11] Y. Loriot, C. Massard, et al., "The Interval from the Last Cycle of Docetaxel-Based Chemotherapy to Progression Is Associated with the Efficacy of Subsequent Docetaxel in Patients with Prostate Cancer," European Journal of Cancer, Vol. 46, No. 10, 2010, pp. 1770-1772. doi:10.1016/j.ejca.2010.04.010

[12] G. Di Lorenzo, C. Buonerba, et al., "Phase II Study of Docetaxel Re-Treatment in Docetaxel-Pretreated Castration-Resistant Prostate Cancer," BJU International, Vol. 107, No. 2, 2011, pp. 234-239. doi:10.1111/j.1464-410X.2010.09498.x

[13] G. Attard, A. H. Reid, et al., "Selective Inhibition of CYP17 with Abiraterone Acetate Is Highly Active in the Treatment of Castration-Resistant Prostate Cancer," Journal of Clinical Oncology, Vol. 27, No. 23, 2009, pp.
3742-3748. doi:10.1200/JCO.2008.20.0642

[14] C. J. Ryan, M. R. Smith, et al., "Phase I Clinical Trial of the CYP17 Inhibitor Abiraterone Acetate Demonstrating Clinical Activity in Patients with Castration-Resistant Prostate Cancer Who Received Prior Ketoconazole Therapy," Journal of Clinical Oncology, Vol. 28, No. 9, 2010, pp. 1481-1488.

[15] D. C. Danila, M. J. Morris, et al., "Phase II Multicenter Study of Abiraterone Acetate plus Prednisone Therapy in Patients with Docetaxel-Treated Castration-Resistant Prostate Cancer," Journal of Clinical Oncology, Vol. 28, No. 9, 2010, pp. 1496-1501.

[16] C. J. Logothetis, E. Basch, et al., "Effect of Abiraterone Acetate and Prednisone Compared with Placebo and Prednisone on Pain Control and Skeletal-Related Events in Patients with Metastatic Castration-Resistant Prostate Cancer: Exploratory Analysis of Data from the COU-AA-301 Randomised Trial," The Lancet Oncology, Vol. 13, No. 12, 2012, pp. 1210-1217. doi:10.1016/S1470-2045(12)70473-4

[17] C. N. Sternberg, A. Molina, et al., "Effect of Abiraterone Acetate on Fatigue in Patients with Metastatic CastrationResistant Prostate Cancer after Docetaxel Chemotherapy," Annals of Oncology, Vol. 24, No. 4, 2012, pp. 1017-1025.

[18] F. Calcagno, T. Nguyen, et al., "Safety and Efficacy of Cabazitaxel in the Docetaxel-Treated Patients with Hormone-Refractory Prostate Cancer," Clinical Medicine Insights: Oncology, Vol. 7, 2013, pp. 1-12.

[19] A. C. Mita, L. J. Denis, et al., "Phase I and Pharmacokinetic Study of XRP6258 (RPR 116258A), a Novel Taxane, Administered as a 1-Hour Infusion Every 3 Weeks in Patients with Advanced Solid Tumors," Clinical Cancer Research, Vol. 15, No. 2, 2009, pp. 723-730. doi:10.1158/1078-0432.CCR-08-0596

[20] J. S. De Bono, S. Oudard, et al., "Prednisone plus Cabazitaxel or Mitoxantrone for Metastatic Castration-Resistant Prostate Cancer Progressing after Docetaxel Treatment: A Randomized Open-Label Trial," The Lancet, Vol. 376, No. 9747, 2010, pp. 1147-1154. doi:10.1016/S0140-6736(10)61389-X

[21] Y. S. Ha, S. Goodin, et al., "Enzalutamide for the Treatment of Castration-Resistant Prostate Cancer," Drugs Today, Vol. 49, No. 1, 2013, pp. 7-13.

[22] H. I. Scher, K. Fizazi, et al., "Increased Survival with Enzalutamide in Prostate Cancer after Chemotherapy," The New England Journal of Medicine, Vol. 367, No. 13, 2012, pp. 1187-1197. doi:10.1056/NEJMoa1207506

[23] A. Berruti, D. Generali, et al., "Enzalutamide in Prostate Cancer after Chemotherapy," The New England Journal of Medicine, Vol. 367, No. 25, 2012, pp. 2448-2449. doi:10.1056/NEJMc1212940

[24] J. Mezynski, C. Pezaro, et al., "Antitumor Activity of Docetaxel Following Treatment with CYP17A1 Inhibitor Abiraterone: Clinical Evidence for Cross-Resistance?" Annals of Oncology, Vol. 23, No. 11, 2012, pp. 2943-2947. doi:10.1093/annonc/mds119

[25] L. Albiges and S. Le Moulec, "Response to Cabazitaxel in 
the Postchemotherapy Setting in CRPC Patients Previously Treated with Docetaxel and Abiraterone Acetate," European Society for Medical Oncology (ESMO), Vienna, 2012.

[26] D. Bianchini, Y. Loriot, et al., "Abiraterone in Patients with Metastatic Castration-Resistant Prostate Cancer Progressing after Docetaxel and MDV3100: A Multicentre Study," European Society for Medical Oncology (ESMO), Vienna, 2012.

[27] K. Noonan and S. Ellard, "Clinical Activity of Abirater- one Acetate (AA) after Progression on mdv3100 in Patients with Metastatic Castration Resistant Prostate Cancer (MCRPC) Abstract," European Society for Medical Oncology (ESMO), Vienna, 2012.

[28] D. Rathkopf, "COU-AA-302, a Randomized Phase 3 Study of Abiraterone Acetate in Metastatic CastrationResistant Prostate Cancer Patients without Prior Chemotherapy," American Society of Clinical Oncology (ASCO), Alexandria, 2013. 